\title{
Entertainment Technology Transfer toward Serious Use
}

\author{
M. Roccetti, G. Marfia, A. Amoroso \\ Computer Science Department \\ University of Bologna \\ 40127 Bologna
}

\{roccetti, marfia, amoroso\}@cs.unibo.it

\author{
C.E. Palazzi \\ Pure and Applied Math Department \\ University of Bologna \\ 35131 Padua \\ cpalazzi@math.unipd.it
}

\author{
D. Maggiorini \\ Computer Science Department \\ University of Milan \\ 20135 Milan \\ dario@dico.unimi.it
}

\begin{abstract}
Since the advent of Web 2.0, online social environments have become common destinations for young people all around the world. In general, entertainment applications and platforms represent the most popular attractive of digital technology. Nonetheless, these virtual environments for users' leisure are currently criticised for their frivolous and selfish aims. One may argue that it is quite disappointing to see huge investments and participation in entertainment technology instead of focusing on health or any other serious issue. Yet, we demonstrate in this paper that technology advancements for leisure applications can easily be transferred toward serious use. To this aim, we discuss three representative examples of entertainment technology transfer we successfully deployed, related to, respectively, sensors for games, social communities and car communication.
\end{abstract}

\section{Categories and Subject Descriptors}

D.3.3 [Personal Computing]: General - games.

\section{General Terms}

Design, Experimentation.

\section{Keywords}

Serious games, entertainment, technology transfer.

\section{INTRODUCTION}

Humans are social beings and, as such, they tend to be attracted by leisure activities and interaction. In this context, entertainment applications are able to attract customers toward new technology, determining the success of new software platforms, which quickly become part of our everyday lives [1].

One representative example is embodied by the commercial success of high-end mobile devices able to ubiquitously communicate and exchange data with each other, which profoundly changed our habits creating what sociologists call homo mobilis: a person, moving around the physical world, while using digital information in a pervasive way.

In this context, social networking is paired with online games as they represent the two most prominent trends emerged in the Web 2.0 era, both started as independent applications and now evolving with cross-contamination. Consider, as an example, the social

Permission to make digital or hard copies of all or part of this work for personal or classroom use is granted without fee provided that copies are not made or distributed for profit or commercial advantage and that copies bear this notice and the full citation on the first page. To copy otherwise, or republish, to post on servers or to redistribute to lists, requires prior specific permission and/or a fee.

DISIO 2012, March 19, 2012, Desenzano, State, Italy.

Copyright 2012 ICST. community of World of Warcraft or, specularly, the popularity of games such as Farmville on Facebook.

In essence, a significant portion of our social interaction now takes place in virtual environments. Nonetheless, these virtual environments for users' interaction are strongly criticized for their frivolousness, arguing that it is quite disappointing to see all this technology and massive participation directed toward selfish and entertaining aims. One would suggest redirecting it toward addressing more crucial problems for humanity [2]. Yet, the technological platforms utilized to support these virtual environments for users' interaction also embody a resource for more serious goals.

This is not a completely new statement; in recent years, social platforms such as social networks and online games have attracted many researchers specialized in various disciplines to tackle their technical challenges. Research in this apparently frivolous topic is often scientifically justified not only with the success of social/gaming applications but also with the promise that their devised solutions could be exported to other, classic, computer science fields [3]. However, one may wonder whether this is just propaganda or there is some real potential for technology transfer. We are strongly in support of the latter and to demonstrate our statement we discuss here some representative examples of technology transfer from frivolous to serious employment; one case study for each among three entertainment related topics: sensors for games, social communities and communication.

The rest of this paper is organized as follows. In Section 2, Section 3, and Section 4 we discuss technology advancement and related technology transfer toward serious employment for sensors for games, social communities and communication, respectively. Finally, Section 5 concludes this paper.

\section{SENSORS FOR GAMES}

During these last ten years, thanks to the tremendous performance improvement of consumer-level hardware, we have been witnessing a rapid growth in the computer game market. These games, once limited to pure video-based entertainment, are now trying to propose new experiences. In particular, one of the most interesting current trends is to exploit the physical movement of the player as a game input.

Indeed, many exergames have been proposed to promote and support fitness, with positive effects on health. Many of these games propose personalized training schedules that are customizable according to height, weight, hearth rate, training history and goals. To foster motivation of players, scoring and rewarding mechanisms are usually implemented as would be in a classic computer game; however, the score can be based on monitored accuracy or effort, thus reinforcing the correct gesture and perseverance. 
This trend has been certainly noticed by big game brands. The Nintendo Wii is the most popular example device enabling physical interactivity between the player and the game. In fact, the game scenario includes onscreen dynamics reproducing gestures and postures of players according to remote control parameters of position, orientation and acceleration [4]. Recently, even Microsoft has demonstrated to have perceived the potential enclosed in new human-machine interactions: the new Natal Project for Xbox uses a webcam pointed toward the user as an input source [5]. The game market is evidently ready to allow programmers and physicians to develop serious games devoted to the rehabilitation of patients.

As the next step, pervasive games represent the bridge between mixed realities and computer games able also to generate augmented games, i.e., computer games that mix digital environments with real world elements [6], [7].

In the meantime, even games for mobile phones have begun to exploit users' real movements for device-human interaction (i.e., rotating the iPhone to have a virtual car steering). It is hence clear that sensors, of course not just movement sensors, represent the current frontier for games.

\subsection{A Game Sensors: Technology Transfer for Wrist Rehabilitation}

We wonder whether this exciting game technology can be used also for some serious purpose. Indeed, the aforementioned game applications embody portable solutions to foster pervasive healthcare systems. Moreover, games involving the sensory-motor system, from reaction times to spatial skills, have already been considered for neural and, in general, functional rehabilitation in everyday tasks [8], [9]. Virtual/augmented reality technology has been used in stroke and motor rehabilitation by investigating the influence of interacting with 3D games. Experiments on motor relearning in a patient suffering from a left arm paresis after stroke demonstrated the improvement in motor patterns, grip strength, and manual dexterity [10]. These technologies clearly embody promising tools for rehabilitation; yet, their capabilities are far from being fully exploited.

As a case study, we have created a game-based tool for wrist rehabilitation: DroidGlove [11]. Wrist rehabilitation is currently accomplished through very expensive and bulky machinery that cannot be moved from hospitals or through exercises that the patient is supposed to perform at home but with no way for the doctor to verify the patient's dedication or performance.

Instead, DroidGlove is a serious game for Android and iPhone platforms that proposes to the users several movement tasks. With DroidGlove, the patient has to go to the doctor carrying along her/his smartphone. After the visit, the doctor shows to the patient a rehabilitation exercise that she/he has to repeat at home. This exercise involves wrist movements that have to be done while holding the smartphone in the hand. The motion sequence identifying rehabilitation movements (i.e., the exergame) can be precisely tracked thanks to sensors present in current smartphones and then the exercise is loaded into the phone memory. To save the exergame's track of movements the doctor can use a PC or record the right movements on the spot by moving the device. A video can be uploaded on the smartphone for the patient to see again the right sequence of movements embodying her/his personalized gametherapy. When at home, or wherever she/he likes, the patient can switch on the smartphone, run the DroidGlove application, see the video, and perform the exergame
(Fig. 1). The application will take care of automatically recording when and how often the exergame has been executed, and how close to the correct (stored) movements the patient performs. A vibration feedback will help the patient in understanding when her/his movements has gone too far from the correct track for that exergame. On the next appointment, beside the usual physical inspection, the doctor will be also able to check the logs in the device so as to supervise the gametherapy.

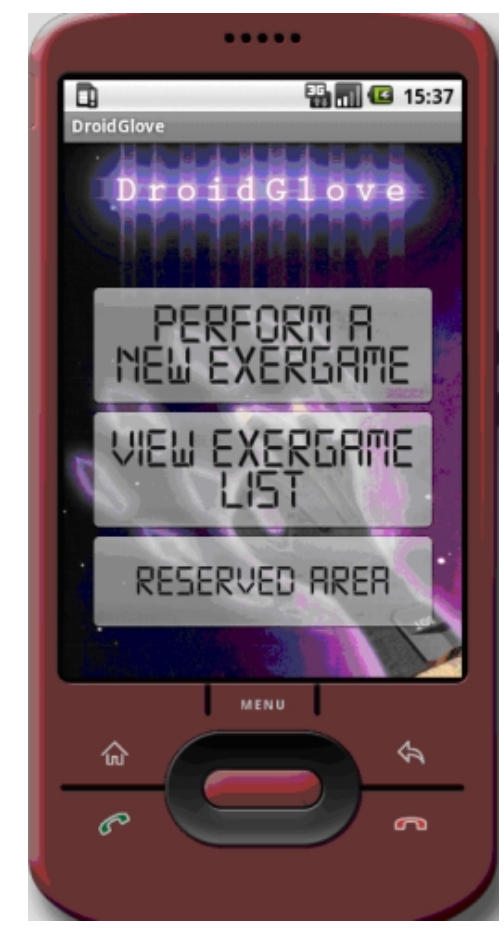

Figure 1. Screenshot of DroidGlove's main menu activity interface.

The user can perform the exercises anytime and anywhere, the smartphone can remind her/him to exercise with a certain frequency, while the gyroscope is utilized to determine the accuracy of the user's movements so as to assign a score (Fig. 2). Both the assigned movement exercises and the accomplished scores can be exchanged in real time between the player and the doctor for a comprehensive supervision. Game technology for exploiting sensed movements is exploited to filter out noise and precisely monitor the patient's movements. Information about performed exercises will be available to the doctor by authentication; the patient will be able to see (or tamper with) the data.

\section{SOCIAL COMMUNITIES}

As a second representative example we consider social communities and their ability to gather millions of users around the world. This ability, mainly dedicated to entertaining social interaction, such as games and social networks, is quickly moving toward mobile users with sensor-equipped smartphones offering them appealing location-based applications.

Think, for instance, to Panoramio, which allows users to take pictures and upload them on the Web with referenced geographic 
coordinates [12]. This way, a map of the world can be navigated at any zoom level to retrieve pictures associated to that location.

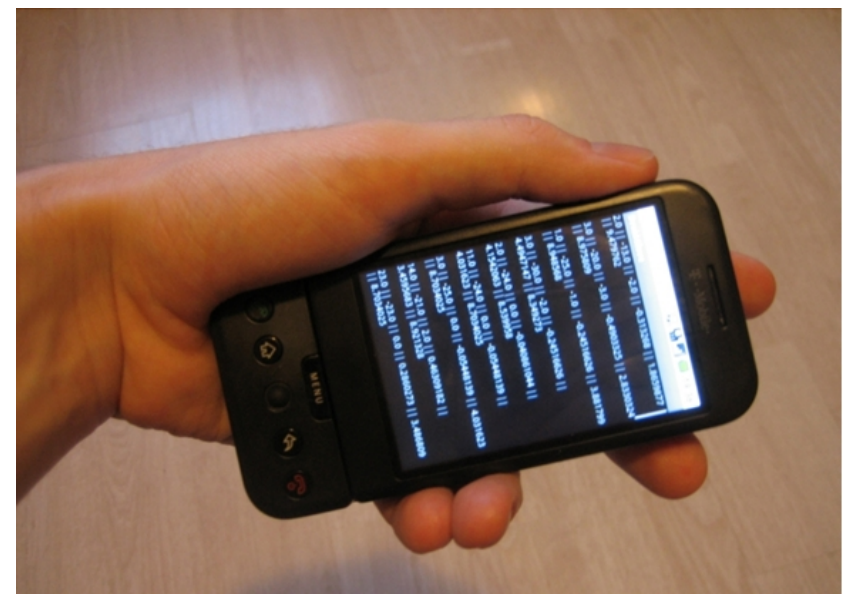

Figure 2. An experimental session with DroidGlove on a HTC G1 smartphone: debug information about acceleration and orientation is reported in real-time on the screen.

Foursquare is an example that allows users to connect with friends and update their location, representing the epicenter of GPS-based applications for a new way to use the phone and live in the city [13]. Connected users can thus be aware of each other's location and meet friends in nearby locations. Furthermore, they can also associate messages to certain locations such as "This restaurant serves the best pizza in town!" or "50\% off last season's snickers", or "we fell in love in this park".

This phenomenon represents a combination among social networking, gaming, and geo-tagging demonstrating how much entertainment is driving technology development in the Web 2.0 era. The success of these kinds of applications is based on two main factors: they reflect new business and socialization needs, and they are very simple to use. The user has just to install the software on the phone, get to a place and then write a message. While doing so, a map is created and shared with friends and acquaintances for information or advice about our favorite (or disliked) places.

These location-based applications, also symbolize the new phase of Web 2.0: the fusion between real and virtual. The online socialization comes into real life and vice versa, thus recomposing the two dimensions of the person. Indeed Web 2.0 applications naturally tend to get better the more people use them, harnessing network effects not only to acquire users, but also to learn from them and build on their contributions by harnessing collective intelligence.

Even better, we can demonstrate in the next subsection that this technology is not just a great innovation for leisure, but its benefits can also be transferred toward more serious applications.

\subsection{Social Communities: Technology Transfer for Accessibility}

To provide a concrete proof that game technology may serve serious purposes we are going to describe the example of a game we designed and implemented, with the aim of delivering a useful service to people with impairments. Before proceeding with this example, we must remind that unfortunately a relevant number of cities, especially those supplied with old road and sidewalk infrastructures, do not provide pedestrians with easy and comfortable ways of accessing their streets, thus requiring walking around or jumping over many obstacles [14].

Solutions have been proposed by researchers to support mobilityimpaired pedestrian through specifically designed route generators [15]-[19]. However, these solutions are based either on the existence of accessibility information related to each road, or on the active participation of users that should create and augment geospatial data. Yet, the crucial problem of gathering accessibility information for every road still remains unsolved.

One possible solution may leverage on those people that, due to a permanent disability or to a temporary illness, are forced to move around one of such cities on a wheelchair. These people create their knowledge on how to move around their neighborhood, or how to reach their job site, from a frustrating trial and error approach that leads them to determine the accessible routes in their area of interest. However, the road accessibility knowledge that is individually created by all the people that walk around a neighborhood can be put to good use for the greater good: all the routes generated by all users can be combined and later used to establish not only the shortest path towards a new destination, but also the most suitable route for people moving on wheelchairs, hence improving city road accessibility. Such service, which we implemented as a serious game, performs the following tasks:

i) The smartphone belonging to each person with impairments records the regular routes of its owner (leftmost part of Fig. 3);

ii) Any user (both with and without impairments) may actively provide the centralized server with a detailed report on some encountered obstacle (e.g., by uploading a photo, video, text or audio fragment) or its own evaluation of its most frequently utilized road segments (rightmost part of Fig. 3);

iii) The centralized server, in turn, while collecting all this information also processes it, identifying the paths that best result to be accessible to wheelchairs;

iv) Any user on a wheelchair that needs to walk through an unfamiliar area can query the centralized server asking for the most accessible route, indicating that she/he will be traversing the area on a wheelchair;

v) The centralized server computes and returns the most suitable route for a person that moves on a wheelchair (Fig. 4, accessible roads are highlighted in purple).

Clearly, the abovementioned steps provide a useful service; yet, it is based on the assumption that people will participate to the community by sending their evaluations of roads' accessibility. It is hence crucial to motivate users in participating in such task. To this aim, a game could be created so as to induce people in performing evaluations of their best known streets. We have hence devised a rewarding scheme, where each player, when providing useful information, wins a number of points. In some sense, our approach extends the Google Image Labeler idea [20].

Yet, rather than simply classifying images, we let participants to classify streets. Upon deciding to deploy such system, we immediately thought that the best way of implementing it was integrating it with the Google Maps service, which already offers a way of easily determining the best routes for cars and pedestrians without disabilities. 

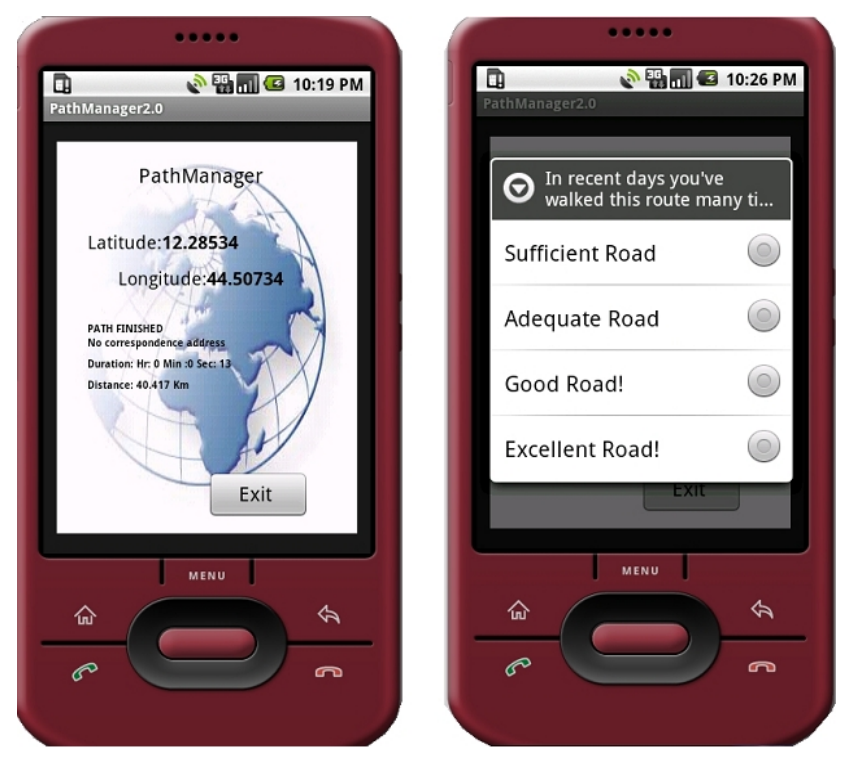

Figure 3. Accessible road management.

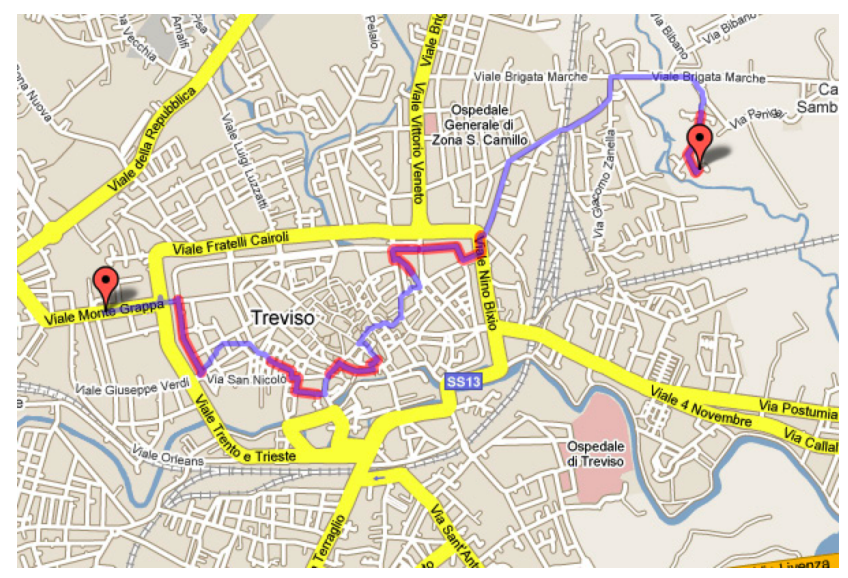

Figure 4. Example of a path obtained by giving high priority to accessible roads.

To summarize, such type of game can serve two purposes. First, it would encourage the provisioning of a service useful to people with impairments. Second, it would increase the players' awareness for problems that are seldom considered. For these reasons, we believe that this and similar approaches can increase the awareness of people for problems that are disregarded by most.

\section{CAR COMMUNICATIONS}

Finally, we want to further strengthen the argument that technologies devised for gaming platforms and technologies devised are substantially equivalent, providing an interesting example. To do this, we here perform the inverse procedure, which is that of starting from a technology that supports a serious application and show how that technology can be put to good use to enable the devise of a computer game, are very closely related and, substantially, equivalent.
Let us, hence, consider the DSRC/IEEE 802.11p technology, which has been thought to provide several new services to vehicle passengers [21], [22]. It is widely agreed that the most prominent application of the DSRC/IEEE 802.11p technology will be that of spreading information regarding traffic accidents. Now, we are interested to understand how this technology could also be useful for networked interactive entertainment within vehicles, thus supporting online games that can be played between passengers, which are traveling on different vehicles. However, very tough are the technical challenges that have to be addressed in order to deploy an interactive online game supported by inter-vehicle communication (IVC).

In fact, one of the most prominent issues in vehicular networks regards the quick, epidemic, and scalable delivery of game events and state updates among all players. Indeed, a feature typically shared by these services is that of having game messages transmitted through a multi-hop, ad-hoc IVC among a group of vehicles (namely a car platoon) covering an area of few kilometres [23]-[25]. The problem in this context is that communications require very tight message delivery time to be effective: typically, under the threshold of a few hundreds of milliseconds [26].

As IVC is wireless and hence shared in nature, it would be highly inefficient to utilize a scheme that, for every game event, generated as many unicast, multi-hop transmissions as the number of players. Rather, the fastest and less resource-consuming way to perform this operation is represented by multi-hop broadcast of messages over the car platoon.

Yet, if no intelligence is applied to the multi-hop broadcasting scheme, any node in the network would simply relay every received message. The consequent explosion in terms of transmitted messages would lead to high congestion, collisions, delays, and even to transmission paralysis of the vehicular network. In some sense, this phenomenon can be seen as a particular instance of what is generally known in MANET's literature as the broadcast storm problem [27].

A solution named Fast Broadcast was recently proposed to provide multi-hop broadcast in a realistic IVC scenario [25]. This solution includes a distributed algorithm for dynamically estimating the transmission range of each vehicle and exploiting it to have the farthest vehicle among those receiving the message in the car platoon to forward it toward the rest of the group. In this way the number of hops a game event has to traverse to cover the whole game platoon, and also the total number of message transmission in the network are reduced to allow a fast broadcasting of each game event.

The basic functioning of Fast Broadcast can be summarized as follows.

i) To achieve a fast multi-hop broadcast of an online game message over a car platoon, Fast Broadcast tries to reduce as much as possible the total amount of transmissions for its propagation; this is achieved by prioritizing farthest vehicles in the message's transmission range in becoming the next forwarder.

ii) To implement this priority system, every sender includes in the sent message its own current transmission range estimation. Receiving vehicles can thus be aware of their position within the sender's transmission range and compute a waiting time which is inversely proportional to the distance from the sender. The vehicle with the smallest waiting time will be the next forwarder. 
iii) Each vehicle continuously computes and updates its transmission range estimation by exploiting data (e.g., position, hearing capability) coming from other vehicles around. To limit the amount of network traffic, these data are included in regular game messages.

iv) Timers are utilized to determine whether, it is time for a vehicle to take charge of forwarding the game message (if no one else was faster).

\subsection{Car Communications: Technology Transfer for Accident Warnings/Online Gaming}

The quick propagation of a message over a car platoon is not a requirement exclusively belonging to online games (or to networked entertainment applications). In vehicular networks, another critical application (with a serious purpose) that has the same need is represented by alert message propagation [28].

In this Subsection we will provide to two different examples which show that a well designed car communication architecture can provide useful support to two different classes of application: inter-car gaming and accident warning systems.

Basically, this application is designed to generate an alert message when a car accident happened and to propagate it around to advise incoming vehicles. As can be seen in Tab. 1, the two aforementioned applications share the same requirements for quick and reliable message delivery to all engaged vehicles [24], [26], [29]-[37].

Table 1. Characterizing properties of the two considered applications.

\begin{tabular}{|c|c|c|}
\hline Feature & Alert Message Broadcast & $\begin{array}{c}\text { Online Interactive } \\
\text { Gaming }\end{array}$ \\
\hline msg sources & one - several cars & many cars \\
\hline msg payload & $\sim 200$ bytes & 〜0bytes \\
\hline $\begin{array}{l}\text { msg rate } \\
\text { generation }\end{array}$ & seldom generation & $10-25$ per second \\
\hline session length & few seconds & several minutes \\
\hline $\begin{array}{l}\text { performance } \\
\text { metric }\end{array}$ & msg delivery time & msg delivery time \\
\hline
\end{tabular}

To compute correct estimation of the transmission range, the Fast Broadcast application needs information, i.e., parameter values, from other vehicles with regularity. This does not mean that messages - and updated parameters - have to be transmitted/received very frequently. Rather, empirical evidence shows that even hearing just one message every $100 \mathrm{~ms}$ is enough for Fast Broadcast to provide a useful estimate of the transmission range. However, certain applications such as, for instance, alert message propagation for traffic safety generate messages only once in a while (minutes). Therefore, to provide readily available transmission range estimation even to these applications, if no message is exchanged in the network for a certain time, Fast Broadcast has to generate sporadic hello messages just for the sake of broadcasting transmission parameters.

We here first report a succinct summary of the results gathered carrying out an experimental study on Fast Broadcast applied to alert message propagation. The main tool utilized for our experiments is the well-known NS-2 simulator (version ns-2.29) with modified MAC layer's parameters to make it behave similar to the IEEE 802.11p [38]. In our simulations, each vehicle generates 10 hello messages, with a payload of $50 \mathrm{~B}$, every second.

We have performed a crash performance evaluation by modelling a car platoon of 30 cars travelling at $32 \mathrm{~m} / \mathrm{s}(115 \mathrm{Km} / \mathrm{h})$, with an inter-vehicular distance of $28.8 \mathrm{~m}$ (the distance travelled in $0.9 \mathrm{~s}$ with a $32 \mathrm{~m} / \mathrm{s}$ speed). At a certain point, the vehicle in front of the platoon has an accident by which its speed is quickly reduced with a deceleration of $8 \mathrm{~m} / \mathrm{s}^{2}$. By braking, following cars decelerate at $4 \mathrm{~m} / \mathrm{s}^{2}$. If a car hits another one in front of it, then both their speeds decelerate at $8 \mathrm{~m} / \mathrm{s}^{2}$ until stopping.

The reaction time of drivers is assumed to be uniformly distributed in the range between $0.75 \mathrm{~s}$ and $1.5 \mathrm{~s}$. Drivers are assumed to be able to see two cars in front of them; they are also assumed to react by braking, after the reaction time, if their car receives an alert message or if one of the two vehicles in front of them brakes. An accident is assumed to happen if the distance between two vehicles becomes smaller than $4 \mathrm{~m}$ (the car's length). Two scenarios are compared: i) no alert messages are implemented, only reaction times, and, ii) alert messages are implemented through our system and all vehicles are able to receive them. The performances achieved in the two scenarios are reported in Fig. 5 and Fig. 6, respectively. The charts show the relative speed between two consecutive cars when they stop or hit each other: an accident occurred when the value is higher than zero and to higher values correspond worst accidents.

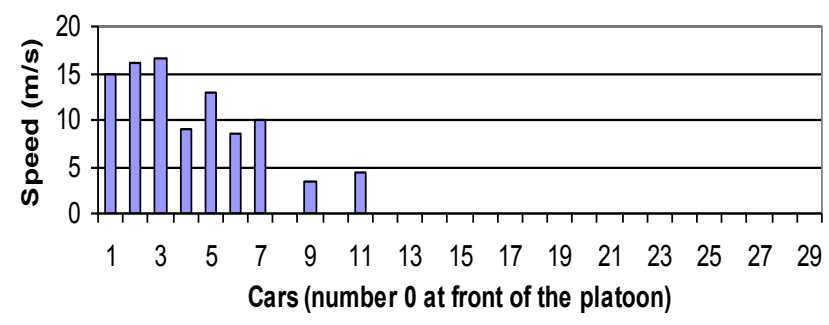

Figure 5. Speed difference between two consecutive cars after stop when employing a regular system with no alert message.

As it is evident, applying the Fast Broadcast scheme reduces the number of vehicles involved in a chain accident. This outcome is not surprising: even few randomly distributed cars receiving the alert message and braking in advance help following cars in anticipating their braking time (they brake as the vehicle in front of them, that received a timely alert message, is braking).

To finalize our argument, we show now as a second step in this discussion that utilizing the same car communication technology discussed above it is possible to implement a rapid delivery of gaming events between vehicles, thus jump over an obstacle that 
could prevent a successful devise of game platform for such type scenarios.

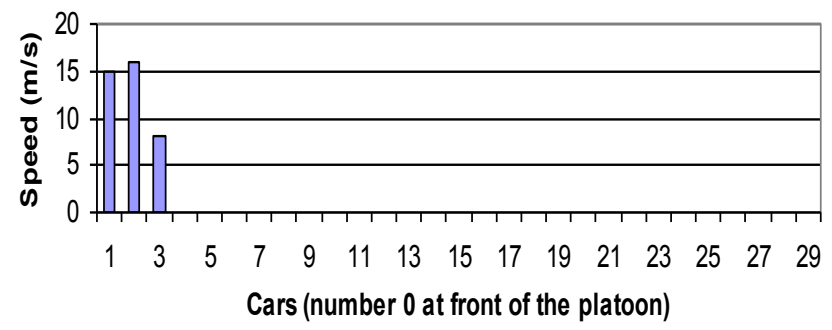

Figure 6. Speed difference between two consecutive cars after stop when employing an alert message application and all vehicles receive the alert message.

We explored such chance assessing how Fast Broadcast is capable of delivering game related messages to an $8-\mathrm{km}$ long platoon of vehicles travelling, as before, at $115 \mathrm{Km} / \mathrm{h}$ and with an intervehicular distance of $28.8 \mathrm{~m}$. The results shown in Fig. 7 reveal that, almost independently from the generation interval of a game event, such algorithm is capable of delivering a message utilizing approximately the same number of hops and, hence, the same number of communication resources. Obviously, the reader should keep in mind that this figure of merit is directly proportional to the delay experienced by a given game event to reach its destination.

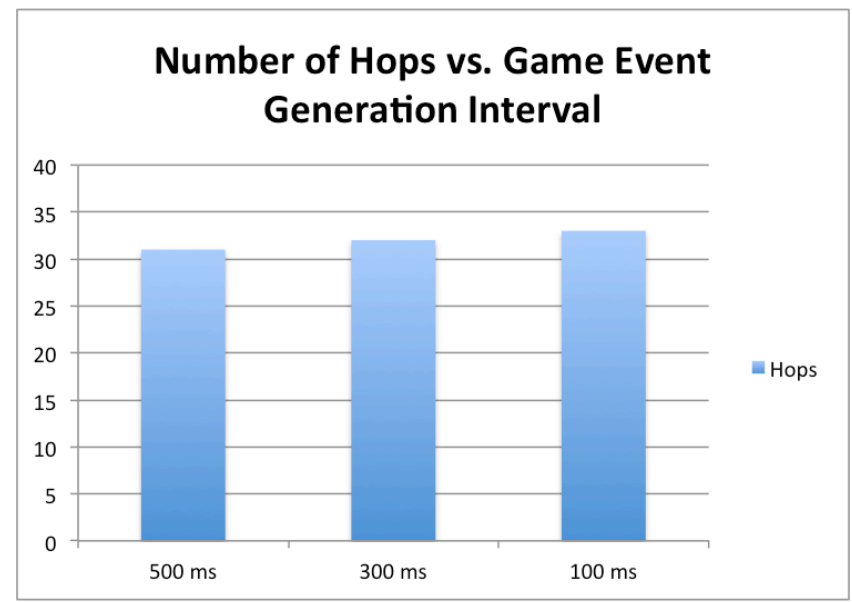

Figure 7. Average number of hops required by a game event to cover an 8-km-long platoon.

Interestingly, what we have just seen is just another proof the very close relation between what is devised for serious applications (e.g., accident prevention) and for leisure ones (e.g., passenger online gaming).

\section{CONCLUSION}

Fun and pleasure play a fundamental role in life. With the proliferation of computer-based entertainment technology, software systems designed for leisure have become a significant part of our common experience. Nonetheless, the great success of leisure application is currently criticized for its frivolous and selfish aims. In this paper we have demonstrated how it is possible to address crucial problems by rerouting the technology developed for games. We have discussed three representative examples. First, we have shown how movement sensors used as new input means for mobile games can be utilized for arm rehabilitation and remote monitoring by physiatrists. Then, we have discussed how location-based social networks based on GPS-endowed smartphones are not necessarily just a leisure application, but they could also be designed to support accessibility in real life for users with impairments. Finally, we have shown that also there is equivalence between the technologies that support gaming and technologies that support other type of applications, taking as a representative example interactive games and accident warning systems. We can hence forget all doubts and welcome innovation in entertainment technology: eventually, the whole society will gain serious benefits from it.

\section{ACKNOWLEDGEMENTS}

Our deepest gratitude goes to Dario Deponti and Lorenzo Teodori for their help in generating prototypes and experiments. We thank the MIUR/PRIN Project ALTERNET for the financial support.

\section{REFERENCES}

[1] Monk, A., Hassenzhal, M., Blythe, M., Reed D., 2009. Funology: Designing Enjoyment. In Proceedings of the ACM Workshop on Human Factors in Computing Systems (CHI'02), Minneapolis, USA

[2] Ferretti, S., Furini, M., Palazzi, C.E., Roccetti, M., Salomoni, P., 2010. WWW recycling for a better world. Communications of the ACM. 53(4):139-143.

[3] Palazzi, C.E., Ferretti, S., Cacciaguerra, S., Roccetti, M., 2005. A RIO-like Technique for Interactivity Loss Avoidance in Fast-Paced Multiplayer Online Games. Computers in Entertainment, ACM, Vol. 3, N. 2, 1-11.

[4] Pearson, P., Bailey, C., 2007. Evaluating the Potential of the Nintendo Wii to Support Disabled Students in Education. In Proceedings of Ascilite, Singapore.

[5] Microsoft Kinect - Project Kinect. Available online : http://www.xbox.com/kinect.

[6] Lindt, I., Ohlenburg, J., Pankoke-Babatz, U., Ghellal, S., Oppermann, L., Adams, M., 2005. Designing Cross Media Games. In 2nd International Workshop on Gaming Applications in Pervasive Computing Environments, Munich, Germany.

[7] Benford, S., Magerkurth, C., Ljungstrand, P., 2005. Bridging the Physical and Digital in Pervasive Gaming. Communications of the ACM. 48(3): 54-57.

[8] Gourlay, D. Lun, K. C., Liya, G., 2000. Virtual Reality and Telemedicine for Home Health Care. Computers and Graphics. 24(5):695-699.

[9] Cameirao, M. S., Bermdez-Badia, S., Duarte Oller, E., Zimmerli, L., Verschure, P. F. M. J., 2007. The Rehabilitation Gaming System: A Virtual Reality Based System for the Evaluation and Rehabilitation of Motor Deficits. In Proceedings of the IEEE Virtual Rehabilitation, Venezia, Italy. 
[10] Broeren, J., Georgsoon, M., Rydmark, M., Stibrant Sunnerhagen, K., 2002. Virtual Reality in Stroke Rehabilitation with the Assistance of Haptics and Telemedicine. In Proceedings of the 4th International Conference on Disability, Virtual Reality and Associated Technologies. Veszprém, Hungary.

[11] Deponti, D., Maggiorini, D., Palazzi, C. E., 2009. DroidGlove: An Android-Based Application for Wrist Rehabilitation. In Proceedings of the IEEE UMSA'09 ICUMT 2009. St. Petersburg, Russia.

[12] Panoramio 2006. Available online: http://www.panoramio.com/

[13] Foursquare 2009. Available online: http://foursquare.com/

[14] Völkel, T., Kühn, R., Weber, G., 2008. Mobility Impaired Pedestrians Are not Cars: Requirements for the Annotation of Geographical Data. In Proceedings of ICCHP'08, Linz, Austria.

[15] Völkel, T., Weber, G., 2008. RouteChecker: Personalized Multicriteria Routing for Mobility Impaired Pedestrians. In Proceedings of the 10th ACM ASSETS'08. Halifax, Canada.

[16] Holone, H., Misund, G., 2008. People Helping Computers Helping People: Navigation for People with Mobility Problems by Sharing Accessibility Annotations. In Proceedings of ICCHP'08. Linz, Austria.

[17] Kasemsuppakorn, P., Karimi, H. A., 2009. Personalised Routing for Wheelchair Navigation. Journal of Location Based Services. 3(1):24-54.

[18] Beale, L., Field, K., Briggs, D., Picton, P., Matthews, H., 2006. Mapping for Wheelchair Users: Route Navigation in Urban Spaces. The Cartographic Journal, Maney Publishing. 43(1):66-81.

[19] Sobek, A., Miller, H., 2006. U-Access: A Web-based System for Routing Pedestrians of Differing Abilities. Journal of Geographical Systems, Springer. 8(3):269-287.

[20] Google Image Labeler 2007. Available online: http://images.google.com/imagelabeler/

[21] DSRC 2005. Dedicated Short Range Communications (DSRC) Home. Available online: http://www.leearmstrong.com/DSRC/DSRCHomeset.htm

[22] Nandan, A., Das, S., Zhou, B. Pau, G., Gerla, M., 2005. AdTorrent: Digital Billboards for Vehicular Networks. In Proceedings of IEEE/ACM V2VCOM. San Diego, CA, USA.

[23] Palazzi, C. E., Roccetti, M., Ferretti, S., Pau, G., Gerla, M., 2007. Online Games on Wheels: Fast Game Event Delivery in Vehicular Ad-hoc Networks. In Proceedings of 3rd IEEE International Workshop on Vehicle-to-Vehicle Communications 2007 (V2VCOM 2007) - IEEE Intelligent Vehicles Symposium 2007, Istanbul, Turkey.

[24] Aoki, M., Fujii, H., 1996. Inter-Vehicle Communication: Technical Issues on Vehicle Control Application. IEEE Communications Magazine. 34(10): 90-93.

[25] Tsugawa, S., 2002. Inter-Vehicle Communications and Their Applications to Intelligent Vehicles: An Overview. In
Proceedings of IEEE Intelligent Vehicle Symposium 2002. Versailles, France.

[26] Gorrieri, R., Roccetti, M., 1993. Towards Performance Evaluation in Process Algebras. In Proceedings of the Third International Conference on Algebraic Methodology and Software Technology (AMAST 1993), Enschede, Netherlands.

[27] Yasseing, M. B., Khaoua, O., Papanastasiou, S., 2005. Performance Evaluation of Flooding in MANETs in the Presence of Multi-Broadcast Traffic. In Proceedings of 11th ICPADS. Fukuoka, Japan.

[28] Palazzi, C. E., Roccetti, M., Ferretti, S., 2010. An InterVehicular Communication Architecture for Safety and Entertainment. IEEE Transactions on Intelligent Transportation Systems. 11(1):90-99.

[29] Fasolo, E., Furiato, R. Zanella, A., 2005. Smart Broadcast Algorithm for Inter-Vehicular Communication. In Proceedings of Wireless Personal Multimedia Communication (WPMC'05). Aalborg, Denmark.

[30] Amoroso, A., Marfia, G., Roccetti, M., 2011. Going Realistic and Optimal: A Distributed Multi-Hop Broadcast Algorithm for Vehicular Safety. Elsevier Computer Networks. 55(10):2504-2519.

[31] Roccetti, M., Marfia, G., Amoroso, A., 2010. An Optimal 1D Vehicular Accident Warning Algorithm for Realistic Scenarios. In Proceedings of the IEEE ISCC'10. Riccione, Italy.

[32] Blum, J.J., Eskandarian A., 2007. A reliable link-layer protocol for robust and scalable intervehicle communications. IEEE Transactions on Intelligent Transportation Systems. 8(1):4-13.

[33] Korkmaz, G., Ekici, E., Ozguner, F., Ozguner, U., 2004 Urban multi-hop broadcast protocol for inter-vehicle communication systems. In Proceedings of the ACM VANET 2004. Philadelphia, PA.

[34] Ye, F., Adams, M., Roy, S., 2008. V2V wireless communication protocol for rear-end collision avoidance on highways. In Proceedings of IEEE Vehi-Mobi 2008. Beijing, China.

[35] Biswas, S., Tatchikou, R., Dion, F., 2006. Vehicle-to-vehicle wireless communication protocols for enhancing highway traffic safety. IEEE Communication Magazine. 44(1):74-82.

[36] Mak, T.K., Laberteaux, K.P., Sengupta, R., 2005. A multichannel VANET providing concurrent safety and commercial services. In Proceedings of the ACM VANET 2005. Cologne, Germany.

[37] Corradini, F., Gorrieri, R., Roccetti, M., 1995. Performance Preorder: Ordering Processes with Respect to Speed. In Proceedings of the 20th International Symposium on Mathematical Foundations of Computer Science (MFCS 1995), Prague, Czech Rep..

[38] Chen, Q., Jiang, D., Taliwal, V., Delgrossi, L., 2006. IEEE 802.11 Based Vehicular Communication Simulation Design for NS-2. In Proceedings of the 3rd ACM VANET, ACM MobiCom 2006. Los Angeles, CA, USA. 